\title{
Niveles de Cromo y Alteraciones de Salud en una Población Expuesta a las Actividades de Curtiembres en Bogotá, Colombia
}

\section{Chromium levels and their relationship with alterations in the health of tannery workers living and working in Bogotá, Colombia}

\author{
Esther Cuberos, Alba I. Rodriguez y Edgar Prieto \\ Departamento de Toxicología, Departamento de Salud Pública. Universidad Nacional de Colom- \\ bia,Bogotá. airodriguezp@unal.edu.co, eprietos@unal.edu.co
}

Recibido 10 Octubre 2008/Enviado para Modificación 13 Diciembre 2008/Aceptado 13 Marzo 2009

\section{RESUMEN}

Objetivo Determinar si los niveles de cromo en orina están más altos de los permitidos y contrastarlos con alteraciones de salud en personas del barrio San Benito en Bogotá. Métodos En una muestra de 827 personas, se cuantificó cromo total en orina como biomarcador de exposición y se contrastó con alteraciones de salud atribuibles a exposición a cromo. La exposición se definió, por la "Ocupación actual" relacionada con la labor en curtiembres. Se definieron dos estratos: "Directamente expuestos" 26 \% y "Potencial alta exposición" $73 \%$.

Resultados Un 6,3\% presentó niveles de cromo >10 ug/L (intervalo de confianza: 4,64$7,96 \%$ ). No se encontraron diferencias estadísticamente significativas entre los dos estratos. El 34,3\% presentó diagnósticos posiblemente atribuibles a la exposición a cromo. El 23,3 \% otorrinolaringológico; 6,5\% dermatológico; 2,9 \% oftalmológico; 1,6 \% cavidad oral y el $65,7 \%$ no relacionados. Se halló asociación entre niveles $>10 \mathrm{ug} / \mathrm{L}$ y residir en la zona (OR 4,94 IC:1,2- 20,7\%). Los resultados sugieren asociación entre ocupación y alteraciones de salud atribuibles a la exposición a compuestos de cromo. Conclusiones Las personas que participan del proceso productivo del cuero tienen un riesgo significativo de presentar hallazgos clínicos posiblemente atribuibles a la exposición a cromo, OR 4,33 (3,12-6,02). La población general se esta viendo expuesta de manera no diferente a aquella con ocupación relacionada con las curtiembres, lo que puede deberse a que los habitantes del sector están en contacto con cromo o compuestos por vías diferentes a la ocupacional, como contaminación ambiental.

Palabras Clave: Curtiembres, industria del cuero, cromo, efectos adversos, toxicidad, exposición ocupacional, cromo toxicidad, cromo orina (fuente: DeCS, BIREME).

\section{ABSTRACT}

Objective Determining whether chromium levels in urine samples were higher than limits and contrasting them with alterations in the health of people living and working in the San Benito neighbourhood of Bogotá.

Methods The total amount of chromium in urine was measured as a biological marker 
of exposure in a sample of 827 people. This was contrasted with health alterations attributed to chromium exposition. Exposure was defined by being whether current economic activity was related to working in a tannery. Two groups were defined: being directly exposed (26\%) and having potentially high exposure $(73 \%)$.

Results $6.3 \%$ presented $>10 \mathrm{ug} / \mathrm{L}$ chromium levels $(4.64 \%$ to $7.96 \%$ confidence interval). No significant statistical differences were found between both groups. $34.3 \%$ presented a diagnosis of possible attribution to chromium exposure, of whom $23.3 \%$ were due to otorhinolaryngologic issues, $6.5 \%$ to dermatological ones, $2.9 \%$ to ophthalmologic ones and $1.6 \%$ to oral cavity issues. The remaining $65.7 \%$ of cases were not related. $>10 \mathrm{ug} / \mathrm{L}$ levels and living in the particular neighbourhood in question were associated (4.94 odds ratio; $1.18 \%-20.69 \% \mathrm{Cl}$ ). The results suggested a connection between economic activity and health alterations due to chromium components.

Conclusions The people involved in producing leather have a significant risk of presenting clinical conditions attributed to chromium exposure (4.33 OR; 3.12-6.02 Cl). San Benito's inhabitants are being exposed to chromium as if they were actually working in a tannery as they are in daily contact with chromium or its components through non work-related activities, such as environmental contamination. Concern at such exposure should lead to further in-depth studies.

Key Words: Tannery, chromium, leather industry, adverse effects, toxicity, occupational exposure, chromium toxicity (source: $M e S H, N L M$ ).

$\mathrm{E}$

1 Cromo $(\mathrm{Cr})$ es un metal, número atómico 24, del grupo VIB de la tabla periódica y peso molecular 51,996. Blanco plateado, brillante, duro y quebradizo, resistente a la corrosión (1). Se encuentra en estados de oxidación $+2,+3,+6$. El estado hexavalente $\mathrm{Cr}(+6)$ el mas importante toxicológicamente, lo presentan los cromatos, dicromatos y el ácido crómico (2). Es un oligoelemento presente en el organismo en forma trivalente $\mathrm{Cr}(+3)$, indispensable en el metabolismo de la glucosa, colesterol, ácidos grasos y cristalino, involucrado en otros múltiples procesos biológicos $(3,4,10)$. Hombre y animales están expuestos al Cr por vía inhalatoria (aire, humo del tabaco), por la piel o por ingestión (productos agrícolas, agua). El mayor peligro profesional ha sido el procesamiento del metal de cromita para producir cromatos $(\mathrm{Cr}+6)$, se encontró que los trabajadores tenían una frecuencia elevada de cáncer pulmonar $(3,6)$. Entre las ocupaciones a riesgo están: minería y trituración, preservación de madera, soldadura, fabricación de cemento, industria de pinturas, industria del cuero, industria fotográfica, industria galvánica, trabajadores de metales y producción de acero inoxidable $(5,3)$.

Toxicología del Cromo. La toxicidad se debe a los derivados $\mathrm{Cr}(+6)$ que, contrariamente a los $\mathrm{Cr}(+3)$ penetran en el organismo por cualquier vía con mayor facilidad. $\mathrm{El} \mathrm{Cr}(+6)$ es considerado carcinógeno del grupo I por la International Agency for Research on Cancer (IARC). El $\mathrm{Cr}(+3)$ no ha sido comprobado como carcinogénico. 
Toxicocinética. El Cr se absorbe por vía oral, respiratoria o dérmica. Se distribuye a nivel de médula ósea, pulmones, ganglios linfáticos, bazo, riñón, e hígado $(9,10,11,14,15)$. La absorción del $\mathrm{Cr}(+3)$ es menor que la del $\mathrm{Cr}(+6)$. $\mathrm{El} \mathrm{Cr}(+3)$ no atraviesa las membranas celulares, uniéndose directamente a la transferrina. $\mathrm{El} \mathrm{Cr}(+6)$ es rápidamente tomado por los eritrocitos e integrado a otras células por el sistema transportador de sulfatos. $(9,10,11,15)$. Metabolismo: $\mathrm{El} \mathrm{Cr}(+6)$ se reduce rápidamente a $(+3)$ intracelularmente a nivel de mitocondrias y el núcleo $(10,11,14,15,16)$. A nivel del citoplasma por reductores intracelulares como el ácido ascórbico, el glutatión, flavo enzimas y riboflavinas. La reducción intracelular genera intermediarios reactivos como $\mathrm{Cr}(+5), \mathrm{Cr}(+4)$ y $\mathrm{Cr}(+3)$, así como radicales libres hidroxilo y oxígeno. Estas formas reactivas del $\mathrm{Cr}$ son susceptibles de alterar el $\mathrm{ADN}(9,10,11,15)$. Eliminación: Por vía renal el $60 \%$, en menor grado por heces (vía biliar), cabello, uñas, leche y sudor (4). En la orina encontramos fundamentalmente $\mathrm{Cr}(+3)$ formando un complejo con el glutation, pues el $\mathrm{Cr}$ (+6) es reducido en gran parte a $\mathrm{Cr}(+3)(1,7,10,15,13,18)$.

Toxicodinamia. Las manifestaciones tóxicas son generalmente atribuidas a los derivados (+6). Entre otras, la acción tóxica se produce por: acción cáustica directa, sensibilización cutánea, citotoxicidad e inflamación de neumocitos, interacción con biomoléculas, el $\mathrm{Cr}(+6)$ induce alteración de cromátidas hermanas, aberraciones Cromosómicas y reacciones cruzadas en la cadena de ADN $(3,10,11,15)$.

Valores permisibles de exposición. Los Valores Límites y en Medios Biológicos han sido propuestos por la American Conference of Governmental Industrial of Hygienists (ACGIH) (15,18), la NIOSH (19), la OSHA y otros. La Secretaria Distrital de Salud de Bogotá reporta valores de referencia para Población General hasta $10 \mu \mathrm{g} / \mathrm{L}$ y para población Expuesta hasta $20 \mu \mathrm{g} / \mathrm{L}$.

Los procesos industriales constituyen la primera fuente de emisión de $\mathrm{Cr}$ al ambiente. La combustión de Carbón y aceite aporta cerca de 1.723 ton. métricas de $\mathrm{Cr}$ por año en emisiones atmosféricas, de este, 0,2 \% es $\mathrm{Cr}(+6)$. En nuestro país existen numerosas industrias que manipulan compuestos de $\mathrm{Cr}$, entre ellas las Curtiembres $(3,7)$. Datos oficiales reportan para el país aproximadamente 800 curtiembres funcionando. El $60 \%$ se ubican en Bogotá y su sabana y de estas, el $90 \%$ en la localidad de Tunjuelito, principalmente en el barrio San Benito donde se concentra el $20 \%$ de la actividad fabril de Tunjuelito (7). El río Tunjuelito recibe 138 vertimientos, con un promedio de 6,7 vertimientos por kilómetro. De la carga de metales pesados arrojada al río, un 79,81\% corresponde a Cr, $19,19 \%$ zinc y el resto de metales $1 \%$. (8). Si bien, se ha descrito la 
exposición a $\mathrm{Cr}$ como un riesgo preponderantemente ocupacional, se considera que los residentes en cercanías a los centros de producción podrían estar expuestos a niveles altos de $\mathrm{Cr}(3,4,5,10)$.

En el Barrio San Benito, Localidad de Tunjuelito se han desarrollado estudios tendientes a disminuir la contaminación por la industria del cuero. En estos estudios no se ha considerado el impacto de la condición actual ambiental en la salud de los habitantes de la zona. Con el presente estudio queremos evidenciar la situación de salud de la población general del Barrio San Benito, diferenciando 2 grupos de expuestos de acuerdo con su relación de ocupación o convivencia (directamente expuestos y potencial alta exposición). En el barrio San Benito se conjugan características que hacen a sus habitantes susceptibles a la exposición crónica al Cr. Se entremezclan actividades industriales, comerciales y zonas residenciales. Hay deficientes condiciones de saneamiento básico, viviendas donde no existe delimitación con la industria. En este trabajo se realizó la determinación de los niveles de $\mathrm{Cr}$ total en orina y la presencia de alteraciones de salud de probable atribución a la exposición a Cr, a través de un estudio poblacional descriptivo observacional de prevalencia con diseño tipo Cross Sectional realizado en el barrio San Benito.

\section{MATERIALES Y METODOS}

Muestra de Estudio. Estudio descriptivo realizado en el 2005, con una muestra de 819 personas. El área geográfica correspondió a la localidad de Tunjuelito en Bogotá. El universo de estudio fue la población del barrio San Benito. La exposición a Cr definida por la Ocupación Actual relacionada o no con el proceso de curtiembres se clasificó en: Directamente expuestos (curtiembres, almacenes, depósitos de químicos) $26 \%$ (215 personas) y de Potencial Alta Exposición (población que no esta en contacto con el tóxico por su ocupación, pero que por la cercanía al proceso industrial, por la ubicación de su residencia cerca de depósitos de residuos y/o materiales en su vivienda pueden incrementar la oportunidad de contacto con el tóxico) con $73 \%$ (604 personas). La unidad de análisis u objeto de observación para este estudio fueron los habitantes y la unidad de muestreo los predios (conglomerado). La muestra se calculó con las siguientes consideraciones a priori: prevalencia esperada de niveles de $\mathrm{Cr}$ en Orina por encima de los limites biológicos permisibles en población general $24 \%$ (20), nivel de Confianza $95 \%$, Error absoluto: $\mathrm{d}=3 \%$.

Con estos valores, el tamaño de muestra fue: $\mathrm{n}=779$ individuos. $\mathrm{Al}$ ajustar por población finita, tomando 16000 habitantes la población total estimada de 
San Benito, $\mathrm{n}=744$ personas y considerando $10 \%$ de pérdida por no participación voluntaria, quedaron 819 personas. La selección se hizo con muestreo aleatorio por conglomerados (personas que comparten predios) con asignación proporcional, 187 predios (51 de expuestos directamente y 137 de potencial alta exposición). Criterio de inclusión: participación voluntaria, firmando el consentimiento informado.

Se cumplió con las normas éticas de investigación en salud, Resolución 8430 de 1993 del Ministerio de Protección Social de Colombia y aprobación del Comité de ética de la Facultad de Medicina de la Universidad Nacional.

Instrumentos o formatos. Consentimiento informado, Examen Médico Toxicológico diseñado para el estudio, Entrega de Recomendaciones. Una vez los individuos aceptaron participar voluntariamente y firmaron el consentimiento se realizó la recolección de la información demográfica, de los antecedentes ocupacionales, la ocupación actual, se realizó la valoración médica y se procedió a la toma de muestras de orina.

Dosificación de Cr en orina. Se recolectaron $100 \mathrm{ml}$ de orina de una micción en frascos de polietileno. Las muestras permanecieron refrigeradas hasta su análisis. El método analítico fue Espectrofotometría de Absorción Atómica con Horno de Grafito, técnica de referencia del instituto de seguridad e higiene del trabajo (INSHT) de Zaragoza España al cual se encuentra adscrito el laboratorio de toxicología de la secretaría Distrital de Salud. El equipo utilizado fue un espectrofotómetro de Absorción atómica UNICAM, modelo 939, con horno de grafito y automuestreador FS9 (21). Rango de detección de la prueba: 0-20 $\mu \mathrm{g} /$ L o ppb (partes por billón) el cual asegura la cuantificación de Cr total tanto en población general como aquella directamente expuesta.

Análisis Estadístico. Para el procesamiento de la información se creo una base de datos en Epi Info 2000, en el análisis se trabajo con Epi 2000, Excel y SAS.

1. Análisis univariado de variables categóricas con estudios de frecuencias, en variables cuantitativas medidas de tendencia central y medidas de dispersión. Los indicadores fueron: Proporción de personas expuestas por su ocupación con niveles por encima de los permitidos. Proporción de personas de potencial alta exposición con niveles por encima de los permitidos. Proporción de personas con hallazgos clínicos positivos por exposición a Cr según lo definido en la literatura internacional. 
2. Análisis bivariado a través de la estimación y comparación de riesgos o Riesgo Relativo (aproximación al riesgo) utilizando tablas y gráficos de frecuencia y modelos de independencia, tablas de contingencia de expuestos Vs. potencialmente de alta exposición con prueba de significancia $\mathrm{Chi}^{2} \mathrm{y}$ OR como medida de asociación para los dos estratos.

\section{RESULTADOS}

Se examinaron 827 personas, de una muestra aleatorizada de predios de la zona. Se encontró una población predominantemente joven con promedio 34 años, el $78 \%$ menor de 50. El grupo de directamente expuestos se encontró entre 21 y 50 años (78 \%); el más joven con 17. En el grupo de Potencial Alta Exposición se encontró $18 \%$ entre 0 a 10 años y en los siguientes decenios de manera más o menos similar. Se distribuyo de manera homogénea en cuanto a sexo. En el grupo de Directamente Expuestos la mayor proporción correspondió a hombres en contraste con el grupo de Potencial Alta Exposición. El nivel educativo fue bajo en ambos grupos, el 93,2 \% solo tiene primaria o secundaria. El 88,4\% posee algún tipo de afiliación al Sistema General de Seguridad Social, 44,3\% al régimen Contributivo, $42,3 \%$ al régimen Subsidiado, y 2,4 \% vinculado. Solo un $12,7 \%$ tiene afiliación a una Administradora de Riesgos Profesionales, muy baja en ambos grupos. La población que labora de una forma u otra con el proceso de curtiembres, solo un $27 \%$ posee vinculación con una ARP. En cuanto a la residencia en la zona, el 64,2 \% de los Expuestos directamente reside en San Benito y el 93,7\% de los Potencialmente Expuestos.

La mayor proporción lleva viviendo entre 16 a 30 años, en contraste con la proporción de los que llevan viviendo menos de 1 año del 3,9\%. Del grupo de Directamente Expuestos el $45 \%$ lleva residiendo en la zona de 16 a 30 años, y en el grupo de Potencial Alta Exposición solo el 27,4 \% se encuentra entre 16 y 30 años. Dentro del grupo de directamente expuestos se apreció que la mayor parte usa parcialmente los elementos de protección personal. Ropa de trabajo (76,6\%), overol $(68,4 \%)$ y botas $(58,9 \%)$.

Evaluación de los niveles de Cr en orina. El 73 \% (604) de la población presentó niveles detectables del metal y un $27 \%$ (223) no presentó niveles por encima del limite de detección. El rango de niveles reportados se encontró entre $0,0130 \mu \mathrm{g} / \mathrm{L}$ y $399 \mu \mathrm{g} / \mathrm{L}$, el promedio fue de $5,30 \mu \mathrm{g} / \mathrm{L}$ una mediana de 1,83 y desviación estándar de 19,6. En el grupo de Directamente Expuestos el $7 \%$ presento niveles por encima de $10 \mathrm{mg} / \mathrm{L}$ y el 3,2 \% por encima de $20 \mathrm{mg} / \mathrm{L}$. En 
el grupo de Potencial Alta Exposición el $9.5 \%$ presento niveles por encima de 10 $\mathrm{mg} / \mathrm{L}$ y por encima de $20 \mathrm{mg} / \mathrm{L}$ el $4,4 \%$.

Evaluación de los hallazgos clínicos atribuibles a la exposición a Cr. Se encontró 284 individuos (34,3\%) con diagnósticos de posible relación y 543 (65,7 $\%)$ con diagnósticos no relacionados, entre ellos, "persona sana". Los hallazgos se clasificaron en: alteraciones dermatológicas, oftalmológicas, de la cavidad oral, y de otorrinolaringología (ORL). La mayor proporción se encontró a nivel de ORL con un 23,3\%, luego las dermatológicas con un 6,5 \%, y las oftalmológicas y cavidad oral con un 2,9 \% y $1,6 \%$ respectivamente. Los diagnósticos mas frecuentes fueron: hipertrofia de cornetes nasales $10,9 \%$, acompañado de inflamación e irritación de la mucosa nasal, 7,9 \%, dermatitis 6,5\%, conjuntivitis 2,9\%.

Al cruzar las diversas variables, con respecto a la asociación entre factores relacionados con la exposición y la presencia de niveles de $\mathrm{Cr}$ en orina, que para nuestro caso se dividió de $0-10 \mu \mathrm{g} / \mathrm{L}$ como nivel normal o aceptado y mayores a $10 \mu \mathrm{g} / \mathrm{L}$ como niveles altos, encontramos los siguientes resultados: No existen diferencias entre los sexos y la presencia de niveles de Cr mayores de $10 \mu \mathrm{g} / \mathrm{L}$. En lo referente a residir en San Benito, se encontró asociación entre detección de $\mathrm{Cr}$ y residir en la zona.

De los datos estratificados según la ocupación actual relacionada o no con curtiembres, se obtuvieron los siguientes resultados: No se encontró asociación entre tiempo de residencia y presencia de niveles de Cr por encima de $10 \mu \mathrm{g} / \mathrm{L}$. Para la variable de riesgo, manipular $\mathrm{Cr}$ en el predio, no se encontró diferencias entre el grupo que si manipula, contra el que no manipula $\mathrm{Cr}$ como factor para presentar niveles elevados.

Al contrastar el tipo de predio contra la presencia de niveles de Cr por encima del valor de referencia aceptado para población general, se agrupo el tipo de predio en 2, así: Predio relacionado con el proceso de curtición (curtiembres, compartidos (curtiembre/habitación), expendio de químicos, local comercial de cueros) y sin relación con las curtiembres (viviendas, local comercial, otros). De acuerdo a esta variable no existe asociación entre la variable tipo de predio y el nivel alto de $\mathrm{Cr}$ en orina en la población del presente estudio.

Se estudiaron algunos hábitos que pueden relacionarse con la elevación de los niveles de Cr, como tabaquismo. Con una p- de 0,14 y un OR de $0,61(0,25-$ 
1,49) no hubo diferencias estadísticamente significativas entre los fumadores de los no fumadores.

Se relacionó el consumo de bebidas alcohólicas, ya que el 83,2 \% de la población que las ingiere describe consumo de cerveza. No se hallaron diferencias estadísticamente significativas entre ambos grupos, OR 0,95 (0,52-1,72), p-valor 0,44 .

En cuanto a las alteraciones de salud de posible atribución a la exposición a Cr se encontró que no existe una evidencia de asociación estadísticamente significativa entre los niveles altos de $\mathrm{Cr}$ y la presencia de los diagnósticos de salud atribuibles a $\mathrm{Cr}$ hallados en nuestro estudio.

Se encontró asociación entre la ocupación actual relacionada con curtiembres y la presencia de alteraciones de salud atribuibles a Cr. OR de 4,33 (3,12-6,02) p-valor 0,000 .

\section{DISCUSIÓN}

$\mathrm{El} \mathrm{Cr}$ es un elemento que se encuentra naturalmente y que puede existir en varias formas químicas y estados de valencia en el ambiente. La biodisponibilidad del Cr, así como la capacidad de atravesar las membranas celulares, constituye un factor importante que determina la toxicidad de los diferentes compuestos. Estudios de la inhalación de mezclas de $\mathrm{Cr}(+3)$ y $(+6)$ en poblaciones ocupacionalmente expuestas soportan la clasificación del $\mathrm{Cr}(+6)$ como un carcinógeno conocido en humanos, pero aporta inadecuados datos para la evaluación de la carcinogenicidad de los compuestos de $\mathrm{Cr}(+3)$.

La exposición ocupacional a $\mathrm{Cr}(+3)$ y $(+6)$ ha sido estudiada en diversos procesos industriales, pero se encuentran pocos datos en la literatura sobre la exposición no ocupacional o poblacional a este elemento. En el presente estudio se analizaron las personas residentes del barrio San Benito, que posee características especiales, como vivir en un barrio predominantemente industrial dedicado a la manufactura y procesamiento del cuero; industria de trayectoria familiar donde se combinan métodos artesanales y técnicos de curtición, no se encuentra delimitada el área industrial de la residencial, existen condiciones ambientales y disposición de residuos industriales deficientes, convirtiendo a su población en personas con un riesgo de exposición al Cr distinto a otras poblaciones. 
En el perfil demográfico de la muestra estudiada, mostró una población joven con un promedio de edad de 34 años, se distribuyo de manera homogénea en cuanto a sexo y con un nivel educativo bajo. El 93,2 \% de la población solo tiene primaria o secundaria. El nivel educativo se comporto de manera muy parecida en ambos grupos, lo mismo que la afiliación y el tipo de afiliación al SGSS.

Llama la atención el porcentaje importante de personas laboralmente relacionadas con el proceso productivo del cuero que tienen una condición de afiliación al régimen subsidiado; se esperaría que teniendo una ocupación tuvieran cobertura por el régimen contributivo y no pertenecieran al subsidiado. Estas cifras permiten dar a conocer a las autoridades de salud pública el comportamiento de la cobertura y la distribución que caracteriza a esta población específica, con el fin de emprender acciones de afiliación, cubrimiento y desarrollo de actividades de promoción y prevención en salud dentro de la concepción del sistema General de Seguridad Social y el de Riesgos Profesionales.

La afiliación al Sistema General de Riesgos Profesionales fue muy baja en ambos grupos. En la población que se encuentra trabajando directamente de una forma u otra con el proceso industrial de las Curtiembres, solo el $27 \%$ tienen vinculación con una ARP. ¿Cómo se están cubriendo las contingencias de Accidente de Trabajo y Enfermedad Profesional así como la prevención y vigilancia de los trabajadores frente a los riesgos relacionados con este trabajo, en el $73 \%$ restante de los trabajadores?

No se encontraron diferencias estadísticamente significativas entre los estratos definidos en razón de la exposición (Potencial Alta Exposición y Directamente Expuestos), lo que lleva a plantear que la población general del barrio San Benito se esta viendo expuesta a compuestos de $\mathrm{Cr}$ de manera no diferente a aquella cuya ocupación esta relacionada con la curtición del cuero, dado que se encontró que en el grupo de Potencial Alta Exposición el 9,5\% presentó niveles por encima de $10 \mu \mathrm{g} / \mathrm{L}$, y el 4,4 \% por encima de $20 \mu \mathrm{g} / \mathrm{L}$, comparado con el grupo de los Directamente Expuestos en el cual el $7 \%$ presento niveles por encima de $10 \mu \mathrm{g} / \mathrm{L}$, y solo el 3,2 \% por encima de $20 \mu \mathrm{g} / \mathrm{L}$. Este resultado puede ser debido a que los habitantes del sector están sometidos al contacto con Cr por otras vías diferentes a la ocupacional, quedando por investigar en futuros estudios, cuales serían las fuentes a la base de esta exposición. Se plantean como posibles, el manejo inadecuado de residuos, la contaminación medioambiental del aire, el agua, los suelos y los alimentos. 
Con los datos obtenidos podemos concluir que la presencia de niveles elevados de $\mathrm{Cr}$ en orina se distribuyo de manera similar en ambos grupos con una proporción ligeramente mayor en el de Potencial alta Exposición. La razón sugerida podría ser por que las personas que se encuentran trabajando en las curtiembres de alguna forma utilizan elementos de protección (76,6 \% utiliza ropa de trabajo). De otro lado, las personas que no laboran en esta producción pero que si comparten vecindad no se protegen. Se evidencia una exposición medioambiental.

Se observó que de las 24 personas que tuvieron niveles de $\mathrm{Cr}$ en orina mayor de $20 \mu \mathrm{g} / \mathrm{L}$, solo 6 personas respondieron encontrarse expuestas a $\mathrm{Cr}$ en su labor actual, 18 personas de las 24 no se encontraban directamente expuestas. Este hallazgo podría confirmar la hipótesis propuesta de otras vías de contaminación.

La concentración de Cr urinario se ha usado con éxito para el monitoreo biológico de personas ocupacionalmente expuestas a niveles mayores de $\mathrm{Cr}$ en ambientes de trabajo, sin embargo, para evaluar la exposición medioambiental a niveles menores, este biomarcador presenta ciertas limitaciones, entre otras: el $\mathrm{Cr}$ urinario representa el $\mathrm{Cr}$ recientemente absorbido, las exposiciones al $\mathrm{Cr}$ deben ocurrir al mismo tiempo, porque la vida media biológica de $\mathrm{Cr}$ en la orina es muy corta (menos de 2 días). La biodisponibilidad y la absorción de rutas menores de exposición como la dérmica y la oral no son fielmente valoradas por este biomarcador. La variabilidad interpersonal es influenciada por la dieta, la ingesta de bebidas, el ejercicio y el hábito del tabaquismo.

Se puede concluir, que existe una asociación estadísticamente significativa entre la ocupación actual relacionada con curtiembres y la presencia de alteraciones de salud relacionadas con la exposición a los compuestos de Cr y que no existe una evidencia de asociación estadísticamente significativa entre niveles altos de $\mathrm{Cr}$ en orina y alteraciones de salud de posible atribución a la exposición al $\mathrm{Cr}$ ni a estar directamente expuesto al proceso de curtiembres.

Nos permitimos afirmar que el impacto de Salud Pública del presente trabajo esta relacionado con el hallazgo que evidenció que tanto la población general, que se consideraba de alguna manera protegida, se esta viendo expuesta al $\mathrm{Cr}$ tanto o mas que la población ocupacionalmente expuesta. Lo anterior nos permite plantear hipótesis relacionadas con otras fuentes de contaminación a nivel ambiental que podrían estar causando los niveles de Cr encontrados en las muestras de orina. Lo anterior nos permite sugerir que en próximos estudios se realice monitoreo ambiental de aguas, alimentos y aire, el comportamiento de las corrientes de aire y porque no, se estudien algunas especies animales. 
En el área del Barrio San Benito se están realizando estudios e intervenciones desarticuladas, unas para el manejo de residuos líquidos y sólidos como manejos puntuales de química e ingeniería en algunos de los curtiembres, otros, desde la secretaría del medio ambiente, limitándose a mediciones extemporáneas medioambientales, en el caso del presente estudio queríamos mostrar efectos en la salud, se recomienda integrar las acciones e intervenciones de avanzar en la solución global del problema y crear un sistema de vigilancia y seguimiento unificado *

Agradecimientos. A los trabajadores y a la población general del Barrio San benito, a Copicur y su gerente quien facilitó el contacto con los curtidores del sector, a los directivos de las empresas participantes. A Susan y a Angélica referentes de medio ambiente y al Gerente del Hospital de Tunjuelito. Fuentes de Financiación: Este proyecto fue financiado por la Universidad Nacional de Colombia y cofinanciado por la Secretaría Distrital de Salud a través del Hospital de Tunjuelito.

Conflictos de interés: Ninguno

\section{REFERENCIAS}

1. Marrett LD, Hartge P, Meigs JW. Bladder Cancer and Occupational Exposure to Leather. $\mathrm{Br} \mathrm{J}$ Ind Med 1986; 43:96-100.

2. Edling C, Kling H, Flodin U. Cancer Mortality Leather Tanners. Br J Ind Med 1986; 43: 494-496.

3. Téllez J, Carvajal RM, Gaitán AM. Aspectos Toxicológicos Relacionados con la Utilización del Cromo en el proceso Productivo de Curtiembres. Revista Facultad de Medicina Universidad Nacional de Colombia 2004;52(1):50-61.

4. Can J. Nasal cancer in leather workers: an occupational disease. Journal of Cancer research and clinical Oncology 1995;121:1-6.

5. Casarett and Doull's Toxicology. The Basic Science of Poisons. 6th ed. New York: McGraw-Hill; 2001.

6. Sunderman JW. Nasal Toxicity, Carcinogenicity and Olfactory Uptake of Metals. Annals of Clinical \& Laboratoy Science, 2001; 31,(1).3-24.

7. Alcaldía Mayor de Bogotá. Diagnostico Local con Participación Social: Localidad de Tunjuelito. Versión Preliminar. Bogotá: s.n.; 2003.

8. Colombia. Instituto de Hidrología Meteorología y Estudios Ambientales (IDEAM), Departamento Técnico Administrativo Medio Ambiente (DAMA). VI Fase de Seguimiento de Efluentes Industriales y Corrientes Superficiales de Bogotá D.C. Bogotá: Imprenta del IDEAM, s.f. 91P. ISBN 958-8067-10-3. 2005.

9. Agency for Toxic Substances and disease Registry (ATSDR). Chromium Toxicity. Case Studies in Environmental Medicine. Course: SS3048 U.S.; 2006.

10. Toxicological review of Trivalent Chromium. In support of Summary Information on the Integrated Risk Information System (IRIS). EPA.; August 1998.

11. Toxicological review of Hexavalent Chromium. In support of Summary Information on the Integrated Risk Information System (IRIS). EPA.; August 1998.

12. IARC-Monographs on the evaluation of carcinogenic risk to humans. Chromium, Nickel and Welding. Vol. 49. IARC, Lyon, France; 1990.

13. Céspedes IJ. Cromo. En: Córdoba PD. Toxicología. $3^{\mathrm{a}}$ ed. s.l.; 1994. pp 228-234. 
14. Chromium VI (hexavalent chromium) entry. Environmental contaminants encyclopedia Compilers/ editors:Roy j. Irwin, national park service. Colorado state university National park service. Water resources divisions, water operations branch. July 11997.

15. Lauwerys RR. Toxicologie Industrielle et intoxications professionnelles. 5th ed. Paris: Masson, 2007. pp 240-254.

16. Ministerio de Trabajo y Seguridad Social. Enfermedades Profesionales. Protocolos para su Diagnóstico. Bogotá : El ministerio, s.f.: 2000 p. 259-270.

17. Grevatt PC. Toxicological Review of Trivalent Chromium. Washington : Environmental Protection Agency; 1988.

18. The American Conference of Governmental Industrial Hygienists. TLVs and BEls 2002. Threshold Limit Values for Chemical Substances and Physical Agents \& Biological Exposure Indices. ACGIH World Wide; 2002.

19. Criteria for a Recommended Standard: Occupational Exposure to Chromium (VI)[1975]HEW (NIOSH) Publication No. 76-129. Presents the criteria and standards for preventing occupational diseases arising from exposure to hexavalent chromium compounds. Revises the chromic acid standard.(Internet). Disponible en http://www.cdc.gov/niosh/pel88/ 7440-47.htm. (consultado: 11 de Julio de 2005).

20. Volney de MC. Estudo dos níveis de exposição e efeitos à saúde por mercúrio metálico em uma população urbana de Poconé, Mato Grosso, Brasil. Cad. Saúde Públ., Rio de Janeiro, janmar, 1996; 12(1):69-77.

21. Instituto Nacional de Seguridad e Higiene en el Trabajo. Protocolo de validación para determinaciones en muestras biológicas (sangre y orina) de interés en Higiene Industrial. MTA/PVIII/98. 1998. 